\title{
EFFECT OF ANISOTROPY OF PROPERTIES OF NICKEL ALLOY ON STRESSES AND PLASTIC DEFORMATIONS IN WELD ZONE
}

\author{
K.A. YUSHCHENKO, E.A. VELIKOIVANENKO, N.O. CHERVYAKOV, G.F. ROSYNKA and N.I. PIVTORAK \\ E.O. Paton Electric Welding Institute, NASU \\ 11 Kazimir Malevich Str., 03680, Kiev, Ukraine. E-mail: office@paton.kiev.ua

\begin{abstract}
A calculation method was used for investigation of the kinetics of stress-strain state in welding of nickel alloy single crystal taking into account anisotropy of its thermophysical and mechanical properties. It is shown that anisotropy of the properties of single crystal promotes for growth of a rate of plastic deformations in temperature interval BTI-II in comparison with isotropic alloy of such type (having polycrystalline structure). The rate of growth of longitudinal plastic deformations in the single crystal in BTI-II temperature interval can approximately 2.0-2.5 times exceed that in the polycrystalline variant of alloy. This should be taken into account in development of corresponding structures and technology of their manufacture. 9 Ref., 3 Tables, 7 Figures.
\end{abstract}

Keywor ds : mathematical modelling, stress-strain state, single crystals, nickel alloys, properties anisotropy

Continuous progress in technology of welding of different materials significantly expands the fields of application of new technologies. Recently, an issue of welding of elements of design from materials with single crystal structure has become relevant. It is known fact that the single crystals are characterized by specific anisotropy of thermophysical as well as mechanical properties [1]. Naturally, that the single crystal being welding can have different reaction of material on heat action of the welding process depending on angles between the welding direction and main crystallographic directions. Thus, various kinetics of temperature fields, stresses and deformations, respectively, can be obtained. Due to known fact that resistance of the single crystals to different fracture types is also determined by level of properties' anisotropy, then development of the effective methods preventing crack formation in single crystal welding requires sufficiently clear ideas on kinetics of the welding stresses and deformations taking into account the anisotropy of thermophysical and mechanical properties. Information from this domain is scarce up to the moment, since corresponding experimental researches require large cost and time expenses. Therefore, it is natural in this connection to use current computer methods realizing corresponding mathematical models [2-4] et al.

In this regard, an aim of this work lied in a calculation investigation of kinetics of stress-strain state in welding of the single crystal of nickel alloy taking into account anisotropy of its thermophysical and mechanical properties.

Unfortunately, using commercial software packages of Ansys or Sysweld type is related with sufficiently serious problem of their reworking for taking into account the anisotropy of properties, and, thus, require engaging corresponding developers of these packages. In order to solve this problem on thermal mechanics of material welding the E.O. Paton Electric Welding Institute many years ago has developed the software package «Weldpredictions», which uses the same mathematical models as in mentioned above commercial packages. Regardless the fact that «Weldpredictions» package has less service programs, its developers currently work at the E.O. Paton Electric Welding Institute, therefore, necessary reworking and specifications of mathematical models, calculation algorithms and computer programs for performance of numerical investigations have been fulfilled by them in sufficiently shot terms.

An essence of the main additions, related with anisotropy of indicated above thermophysical and mechanical properties applicable to nickel alloy single crystal:

heat conductance coefficient $\lambda_{n}$ determining the components of a vector of heat flow

$$
f_{n}=\lambda_{n} \frac{\partial T}{\partial n}, \quad(n=x, y, z)
$$

in arbitrary point $x, y, z$ of heat conducting medium, according to [1] is the second-rank tensor, in which only diagonal elements differ from zero

$$
\lambda_{n}=\left\|\begin{array}{ccc}
\lambda_{x x} & 0 & 0 \\
0 & \lambda_{y y} & 0 \\
0 & 0 & \lambda_{z z}
\end{array}\right\|
$$

(in accordance with Fourier heat conduction theory).

Sum of such tensor on [1]

$$
\lambda_{x x}+\lambda_{y y}+\lambda_{z z}=3 \lambda_{\mathrm{av}},
$$

where $\lambda_{\mathrm{av}}$ is the average value of heat conductance coefficient.

For single crystal with typical cubic lattice the typical crystallographic directions (Figure 1) are: [001] — along the boundaries; [011] — along small diagonals and [111] — along large diagonals. 
If $x, y, z$ coordinate system matches with [001] directions, then $\lambda_{x x}=\lambda_{y y}=\lambda_{z z}=\lambda_{\mathrm{av}}=\lambda_{001}$.

If axis $x$ with [011] direction forms an angle $\omega$, and axis $z$ matches with direction [001], then $\lambda_{z z}=\lambda_{001}$, and $\lambda_{x x}$ and $\lambda_{y y}$ are determined by dependencies

$$
\begin{gathered}
\lambda_{x x}=\lambda_{011} \cos ^{2} \omega+\left(2 \lambda_{001}-\lambda_{011}\right) \sin ^{2} \omega, \\
\lambda_{y y}=2 \lambda_{001}-\lambda_{x x}=2 \lambda_{001} \cos ^{2} \omega-\lambda_{011} \cos 2 \omega .
\end{gathered}
$$

If axis $x$ makes an angle $\rho$ with direction [111], and plane $x O y$ comes through two small diagonals and cube edges, then $\lambda_{z z}=3 \lambda_{\mathrm{av}}-\lambda_{001}-\lambda_{011}=2 \lambda_{001}-$ $\lambda_{011}$ and respectively

$$
\begin{gathered}
\lambda_{x x}(\rho)=\lambda_{111} \cos ^{2} \rho+ \\
+\left(3 \lambda_{001}-2 \lambda_{001}+\lambda_{011}-\lambda_{111}\right) \sin ^{2} \rho= \\
=\lambda_{111} \cos ^{2} \rho+\left(\lambda_{001}+\lambda_{011}-\lambda_{111}\right) \sin ^{2} \rho .
\end{gathered}
$$

More general positions of the axis $x$ (axis of movement of welding heat source) relatively to the main crystallographic planes (directions) are also possible. However, we will use variant (4) for simplicity.

Already at this it can be shown that orthotropy of $\lambda$ value according to (4) can result in noticeable effects in temperature fields. Mentioned above is demonstrated below by the example of welding heating with movable power source (arc in argon medium, $I=$ $=95 \mathrm{~A}, U=10.5 \mathrm{~V}, v_{\mathrm{w}}=8.5 \mathrm{~m} / \mathrm{h}$ ) of fine plate from the single crystal of nickel alloy. The average thermophysical $(c \gamma, \lambda, \alpha)$ and mechanical $\left(E, \sigma_{\mathrm{v}}\right)$ properties used in calculations, are given in Table 1. Size of plate $L_{x}=85 \mathrm{~mm}, L_{y}=40 \mathrm{~mm}, \delta=3 \mathrm{~mm}$.

Figure 2 shows the temperature fields close to weld pool moving along axis $x$ depending on angle $\omega=$

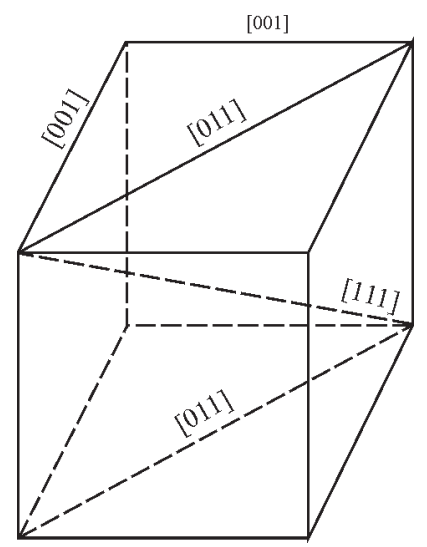

Figure 1. Main crystallographic directions in single crystal with cubic lattice

$=0-\pi / 2$ under condition that $\lambda_{011}=1.5 \lambda_{\text {av }}$. Scalar value $c \gamma$ was taken constant and equal $c \gamma_{\mathrm{av}}(T)$ on Table 1.

It can be seen from these data that the temperature fields can significantly vary depending on angles between the welding direction and crystallographic directions of single crystal at value $\lambda$ indicated anisotropy.

For variant $\omega=\pi / 4$, when $\lambda_{x x}=\lambda_{y y}=\lambda_{z z}=\lambda_{\text {av }}$ the calculation results agree well with experimental data $\lambda(T)$ and $c \gamma(T)$ according to Table 1 under normal-volumetric distribution of effective power of the source, when coefficient of efficiency $\eta_{\mathrm{s}}=0.6$, and coefficients of concentration of source $K_{y}=K_{x}=0.25 \mathrm{~mm}^{-2}$, $K_{z}=0.8 \mathrm{~mm}^{-2}$.

It is important to carry out comparative evaluation of effect of studied anisotropy of $\lambda$ on kinetics of deformations and stresses in typical points, critical from point of view of technological strength. From

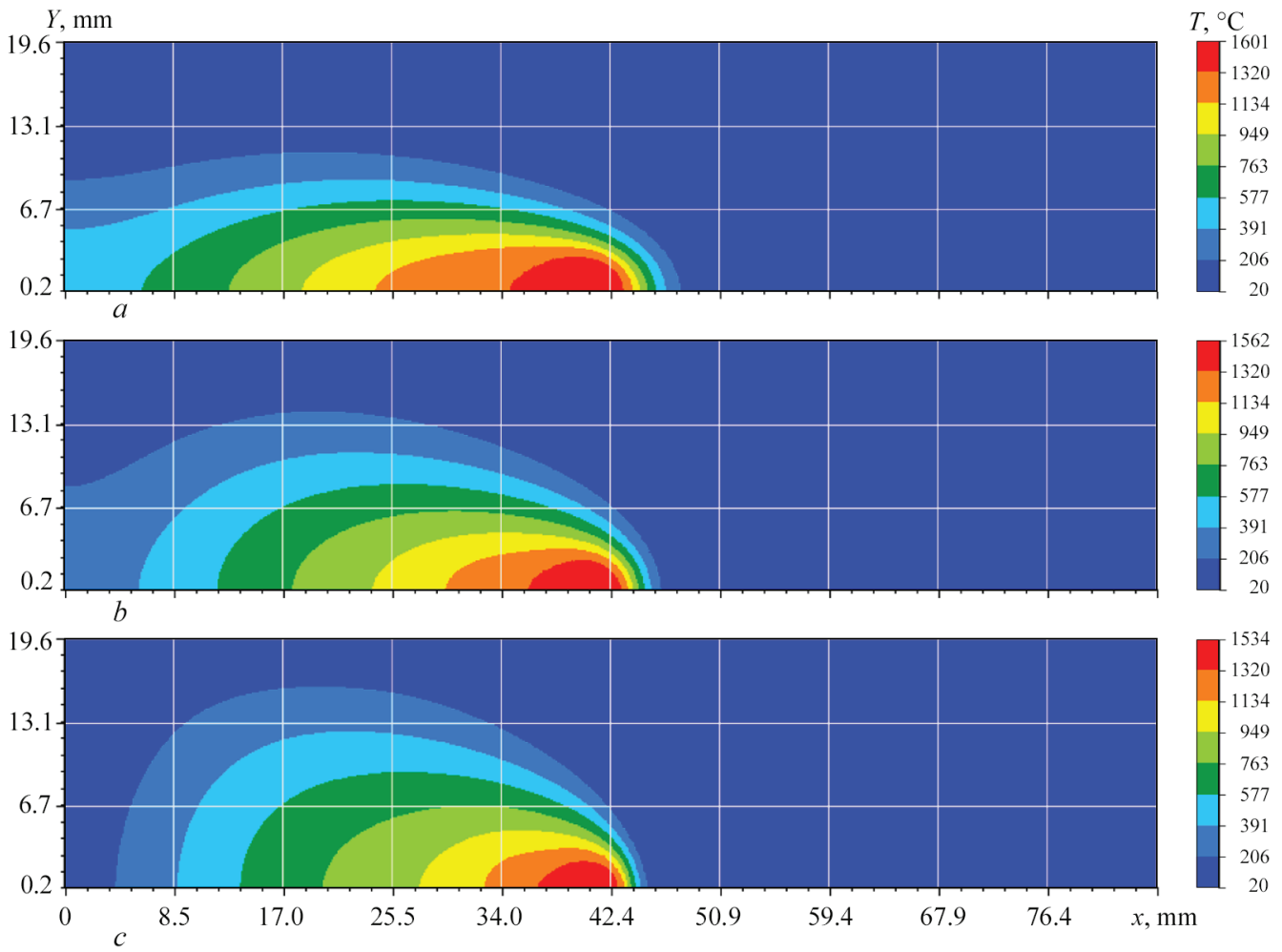

Figure 2. Temperature field close to weld pool: $a$ - at $\omega=0$ (2.85 mm pool width, $9.4 \mathrm{~mm}$ length); $b-\omega=\pi / 4(2.55 \mathrm{~mm}$ pool width, $7.7 \mathrm{~mm}$ length); $c-\omega=\pi / 2(2.35 \mathrm{~mm}$ pool width, $6.4 \mathrm{~mm}$ length $)$ 
Table 1. Average thermophysical and mechanical characteristics of studied alloy

\begin{tabular}{|c|c|c|c|c|c|}
\hline$T,{ }^{\circ} \mathrm{C}$ & $\mathrm{c} \gamma, \mathrm{cal} /\left(\mathrm{mm}^{3 .}{ }^{\circ} \mathrm{C}\right)$ & $\lambda, \mathrm{cal} /\left(\mathrm{mm} \cdot \mathrm{s} \cdot{ }^{\circ} \mathrm{C}\right)$ & $E \cdot 10^{-5}, \mathrm{MPa}$ & $\sigma_{y}, \mathrm{MPa}$ & $\alpha \cdot 10^{4}, 1 /{ }^{\circ} \mathrm{C}$ \\
\hline 50 & 0.0008063 & 0.0018 & 1.838 & 930 & 0.110 \\
\hline 100 & 0.0007797 & 0.0019 & 1.838 & 930 & 0.114 \\
\hline 200 & 0.0008506 & 0.0023 & 1.838 & 875 & 0.122 \\
\hline 300 & 0.0008417 & 0.0024 & 1.838 & 875 & 0.140 \\
\hline 400 & 0.0008594 & 0.0027 & 1.838 & 825 & 0.143 \\
\hline 500 & 0.0008683 & 0.0030 & 1.838 & 800 & 0.150 \\
\hline 600 & 0.0008771 & 0.0036 & 1.838 & 800 & 0.162 \\
\hline 700 & 0.0008949 & 0.0036 & 1.838 & 800 & 0.185 \\
\hline 800 & 0.0009746 & 0.0041 & 1.838 & 790 & 0.240 \\
\hline 900 & 0.0010366 & 0.0044 & 1.838 & 650 & 0.310 \\
\hline 1000 & 0.0010898 & 0.0048 & 1.585 & 375 & 0.380 \\
\hline 1100 & 0.0012138 & 0.0055 & 0.529 & 125 & 0.410 \\
\hline 1200 & 0.0012138 & 0.0055 & 0.271 & 25 & 0.390 \\
\hline
\end{tabular}

this point of view and applicable to nickel alloys, the points close to the fusion line are critical for risk of formation of transverse hot cracks in corresponding alloy brittleness temperature interval BTI [5]. Evaluation of weldability of this alloy on Varestraint-Test procedure showed that the material has two BTI:

BTI-I $\approx 1350-1250^{\circ} \mathrm{C}, \varepsilon_{\mathrm{cr}} \approx 0.38 \%$;

BTI-II $\approx 1050-800^{\circ} \mathrm{C}, \varepsilon_{\mathrm{cr}} \approx 0.10 \%$.

The positive increments of plastic deformations above $\varepsilon_{\text {cr }}$ at tensile normal stresses in corresponding direction will promote crack formation.

The calculations, which were carried with the help of «Weldpredictions» package, for points located in the boundary from fusion line $\Delta y=-0.5 \mathrm{~mm}$ (point 1 ), $\Delta y$ $=0.5 \mathrm{~mm}$ (point 2) and $\Delta y=1.5 \mathrm{~mm}$ (point 3) (Figure 3 ) at different $\omega=0-\pi / 2$ showed the level of effect of studied anisotropy of $\lambda$ on kinetics of changes of corresponding plastic deformations $\Delta \varepsilon_{x x}^{p}$ and stresses in points $1-3$ depending on temperature in these points.

Figures 4 and 5 show such results for point 2 , and Table 2 gives the increments $\Delta \varepsilon_{x x}^{p}$ and the limits of change of $\sigma_{x x}$ stresses in the BTI-II for all studied points $1-3$.

It can be seen that anisotropy of $\lambda$, corresponding to $\omega=0$ and $\omega=\pi / 2$, in comparison with $\omega=\pi / 4$, when anisotropy does not become apparent, results in noticeable change of rate of plastic deformation increments in BTI-II.

Possible anisotropy of a coefficient of relative temperature elongation $\alpha$ in comparisons with $\alpha_{\text {av }}$ from Table 1 can also have specific effect on the rate of plastic deformations in the BTI zone.

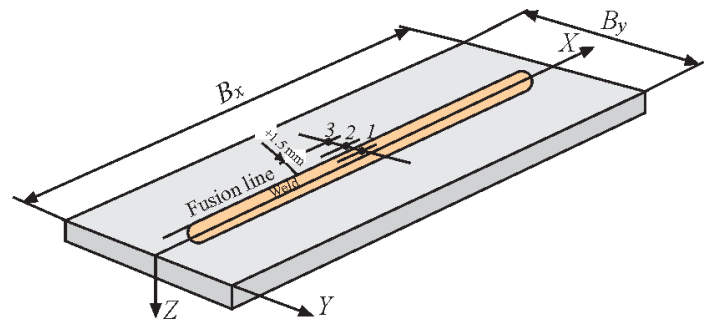

Figure 3. Position of studied points on specimen $\left(B_{x}-\right.$ specimen length, $B_{y}$ - specimen width, 1 - point in weld at $-0.5 \mathrm{~mm}$ distance from fusion line; 2,3 - point in HAZ at 0.5 and $1.5 \mathrm{~mm}$ distance from fusion line, respectively)
Since $\alpha$ value determines the components of a vector of normal temperature deformations in a single crystal, then description of its anisotropic changes similar to heat conduction coefficient can be done using dependence (4).

Data given in Table 2 show an effect of $\omega$ angle at $\alpha_{011}=1.5 \alpha_{001}$ and $\alpha_{001}=\alpha_{\text {av }}$ on Table 1. It can be seen that anisotropy of $\alpha$ similar to anisotropy of $\lambda$ results qualitatively in similar effect on rate of $\Delta \varepsilon_{x x}^{p}$ deformations in the BTI-II.

Thus, anisotropy of $\lambda$ and $\alpha$ in the single crystal depending on angle $\omega$ develops some orthotropy in mathematical description of temperature field and temperature deformations with periodicity on angles $\omega$ and $\rho$ in $\pi / 2$. Somewhat another effect can be related with anisotropy of mechanical characteristics (elasticity modulus E and yield strength $\sigma_{y}$ ).

Tables 3 and Figure 6 give the experimental data relatively to value of elasticity modulus $E(T)$ of the single crystals of nickel alloys on different sources. It can be seen that sufficiently noticeable anisotropy of $\omega$ and $\rho$ with $\pi / 4$ period takes place on this characteristic.

The results of application of these data for the considered calculation model at $\rho=0$ and different $\omega$ are given in Table 2. It follows from them that in comparison with $\omega=\pi / 4$ state (isotropy state) reduction of $\omega$ results in rise of $E$ and respectively increase of plastic deformations in the BTI-II zone.

Experimental data from work [6] relatively to anisotropy of yield strength $\sigma_{0.2}=\sigma_{y}(T)$ in Figure 7 for single crystal PWA1480 show that anisotropy is sufficiently large in different deformation planes. However, it is impossible to take it into account based on the Mises yield criterion, since under this condition plastic flow occurs in an elementary volume without specification of flow plane. The Tresca yield criterion is more reasonable for consideration of the plastic flow plane. For it a yield surface can be presented through functional $f[3,7]$ :

where $\Theta=\pi / 4-\omega$. 

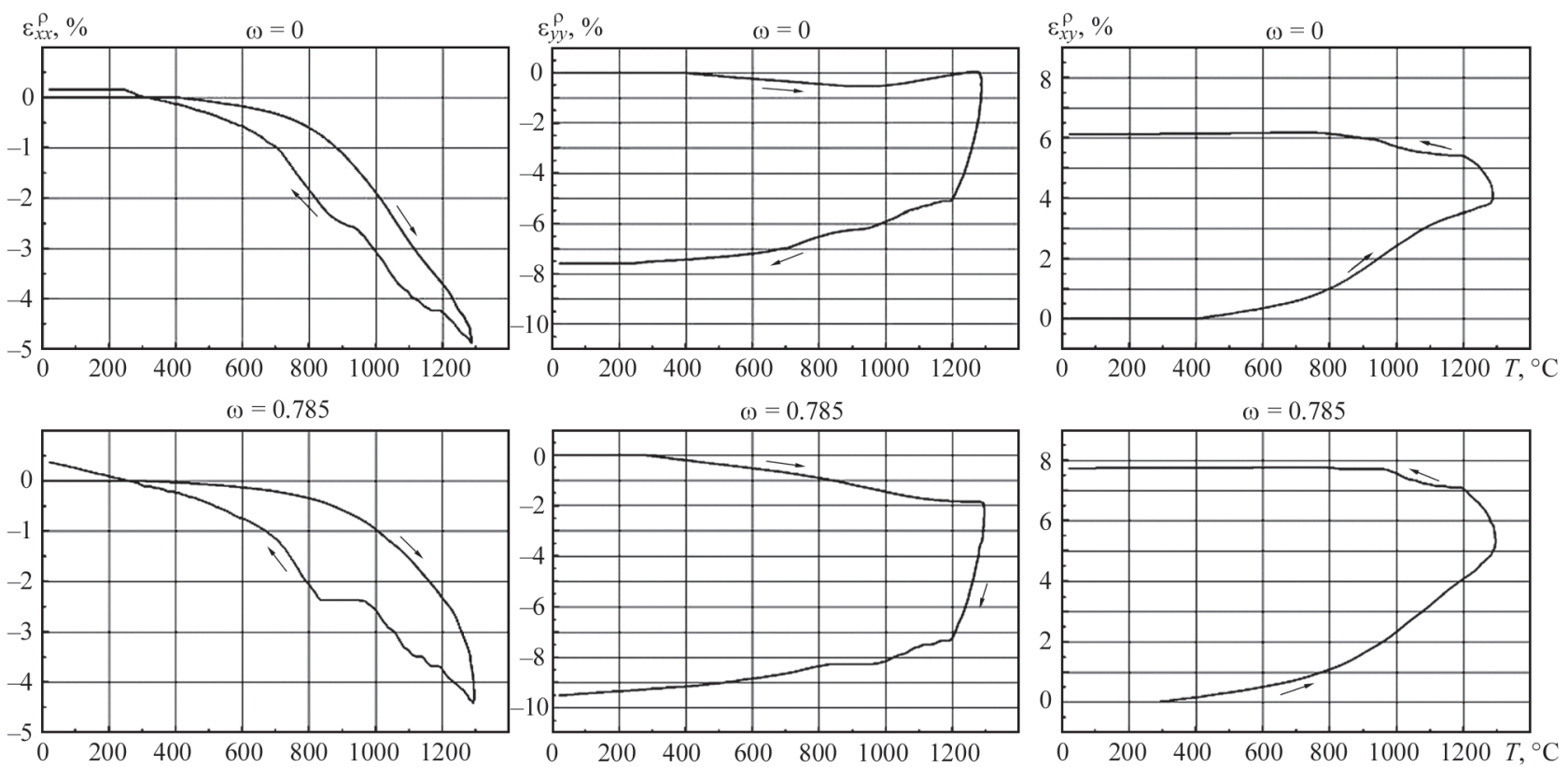

$\omega=1.57$

$\omega=1.57$

$\omega=1.57$
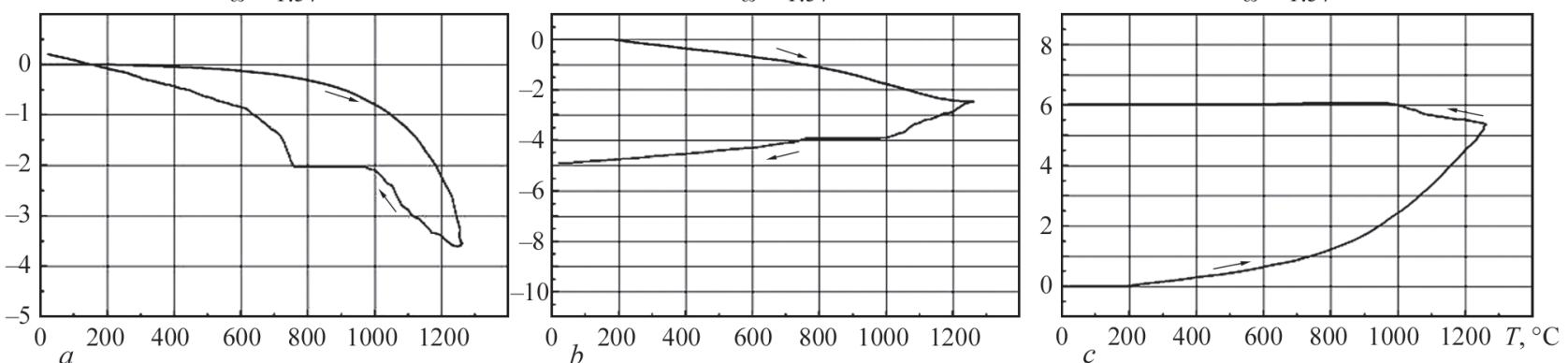

Figure 4. Kinetics of change of plastic deformations: $a-\Delta \varepsilon_{x x}^{p}, b-\Delta \varepsilon_{y y}^{p}, c-\Delta \varepsilon_{x y}^{p}$ from temperature in point $2(\Delta y=0.5 \mathrm{~mm})$ taking into account anisotropy of $\lambda$ for different $\omega$

The maximum tangential stresses $\tau_{\max }$ are determined in form of [8]

$$
\tau_{\max }=\max \left\{\begin{array}{c}
\frac{1}{2} \sqrt{\left(\sigma_{x x}-\sigma_{y y}\right)^{2}+4 \sigma_{x y}^{2}} \\
\frac{\sigma_{x x}+\sigma_{y y}}{4}+\frac{1}{4} \sqrt{\left(\sigma_{x x}-\sigma_{y y}\right)^{2}+4 \sigma_{x y}^{2}}
\end{array}\right.
$$

and act in a slip plane, position of which is determined by condition if:

$$
\sigma=\frac{\sigma_{x x}+\sigma_{y y}}{2}>0
$$

then a slip plane is located at $\pi / 4$ angle to axis $z$ and at $\varphi$ angle to axis $x$, where $\varphi$ is determined by condition

$$
\operatorname{tg} 2 \varphi=\frac{2 \sigma_{x y}}{\sigma_{x x}-\sigma_{y y}} .
$$

\begin{tabular}{|c|c|c|c|c|c|c|c|}
\hline \multirow{2}{*}{\multicolumn{2}{|c|}{ Characteristics of variant }} & \multicolumn{2}{|c|}{ Point $1(\Delta y=-0.5 \mathrm{~mm})$} & \multicolumn{2}{|c|}{ Point $2(\Delta y=0.5 \mathrm{~mm})$} & \multicolumn{2}{|c|}{ Point $3(\Delta y=1.5 \mathrm{~mm})$} \\
\hline & & \multirow{2}{*}{$\frac{\Delta \varepsilon_{x x}^{p}, \%}{0.8}$} & \multirow{2}{*}{$\begin{array}{c}\sigma_{x x}, \mathrm{MPa} \\
100-900\end{array}$} & \multirow{2}{*}{$\frac{\Delta \varepsilon_{x x}^{p}, \%}{0.9}$} & \multirow{2}{*}{$\begin{array}{c}\sigma_{x x}, \mathrm{MPa} \\
100-900\end{array}$} & \multirow{2}{*}{$\frac{\Delta \varepsilon_{x x}^{p}, \%}{17}$} & \multirow{3}{*}{$\begin{array}{c}\sigma_{x x}, \mathrm{MPa} \\
-200-850 \\
-200-800\end{array}$} \\
\hline Main. Complete an & ppy & & & & & & \\
\hline \multirow{3}{*}{ Anisotropy of $\lambda$} & $\omega=0$ & 1.7 & $100-800$ & 1.5 & $100-900$ & 1.8 & \\
\hline & $\omega=\pi / 4$ & 0.8 & $100-900$ & 0.9 & $100-900$ & 1.7 & $-200-850$ \\
\hline & $\omega=\pi / 2$ & 0.4 & $100-900$ & 0.6 & $100-900$ & 1.4 & $-200-800$ \\
\hline \multirow{3}{*}{ Anisotropy of $\alpha$} & $\omega=0$ & 1.5 & $180-900$ & 2.0 & $150-900$ & 2.5 & $-200-880$ \\
\hline & $\omega=\pi / 4$ & 0.8 & $180-900$ & 0.9 & $150-900$ & 1.7 & $-200-850$ \\
\hline & $\omega=\pi / 2$ & 0.3 & $180-900$ & 0.3 & $150-900$ & 1.0 & $-200-800$ \\
\hline \multirow{3}{*}{ Anisotropy of $E$} & $\omega=0$ & 0.2 & $100-850$ & 0.4 & $200-850$ & 1.3 & $-200-800$ \\
\hline & $\omega=\pi / 8$ & 0.5 & $180-850$ & 0.7 & $180-850$ & 1.4 & $-200-800$ \\
\hline & $\omega=\pi / 4$ & 0.8 & $180-900$ & 0.9 & $200-900$ & 1.7 & $-200-800$ \\
\hline \multirow{3}{*}{ Anisotropy of $\sigma_{y}$} & $\omega=0$ & 1.0 & $100-1000$ & 1.4 & $100-1000$ & 1.5 & $-200-1000$ \\
\hline & $\omega=\pi / 8$ & 0.9 & $100-1000$ & 1.3 & $100-1000$ & 1.4 & $-200-1000$ \\
\hline & $\omega=\pi / 4$ & 0.8 & $100-1000$ & 0.9 & $100-1000$ & 1.7 & $-200-1000$ \\
\hline \multirow{3}{*}{ Anisotropy of $\lambda, \alpha, E, \sigma_{\mathrm{y}}$} & $\omega=0$ & 2.0 & $200-1000$ & 2.5 & $200-1000$ & 2.5 & $-200-1000$ \\
\hline & $\omega=\pi / 8$ & 1.6 & $200-1000$ & 3.5 & $200-1000$ & 4.0 & $0-1000$ \\
\hline & $\omega=\pi / 4$ & 0.8 & $200-1000$ & 0.9 & $200-1000$ & 1.7 & $-250-1000$ \\
\hline
\end{tabular}

Table 2. Comparison of deformations $\Delta \varepsilon_{x x}^{p}$ and $\sigma_{x x}$ stresses in BTI-II $(1050-800){ }^{\circ} \mathrm{C}$ 

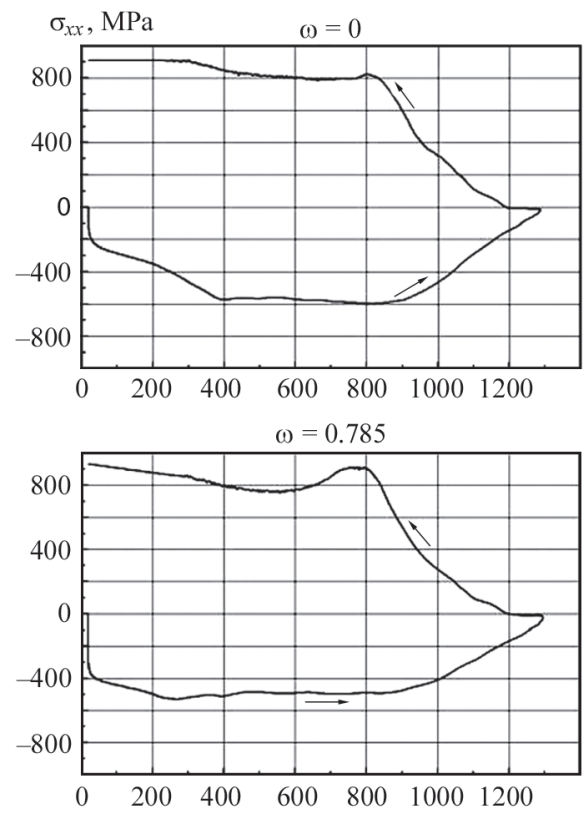

$\omega=1.57$

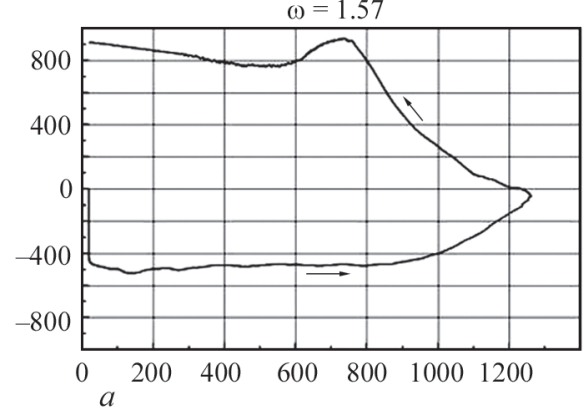

$\sigma_{y y}, \mathrm{MPa}$
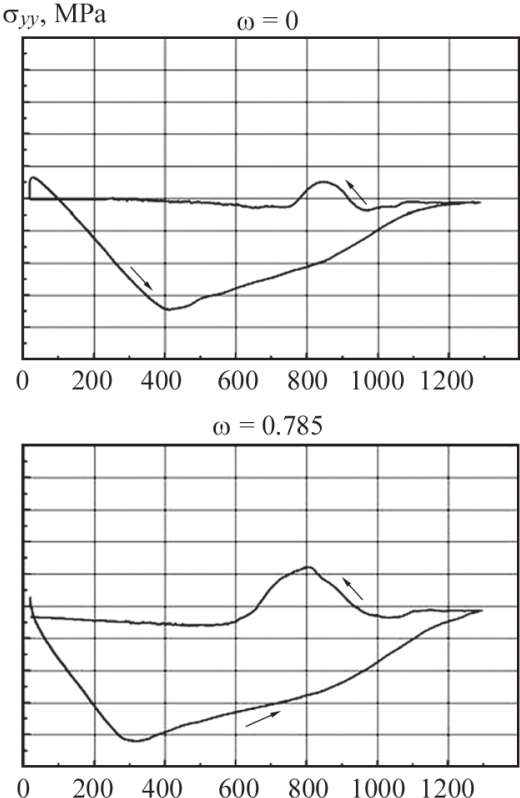

$\omega=1.57$

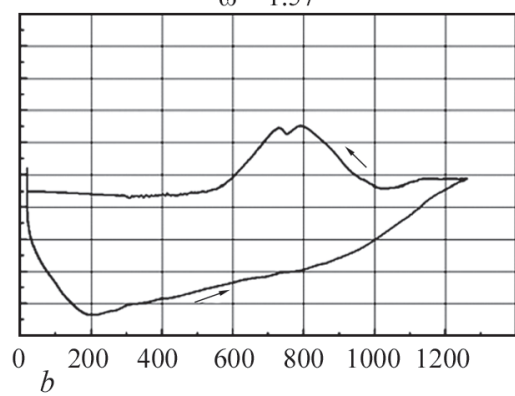

$\sigma_{x y}, \mathrm{MPa}$
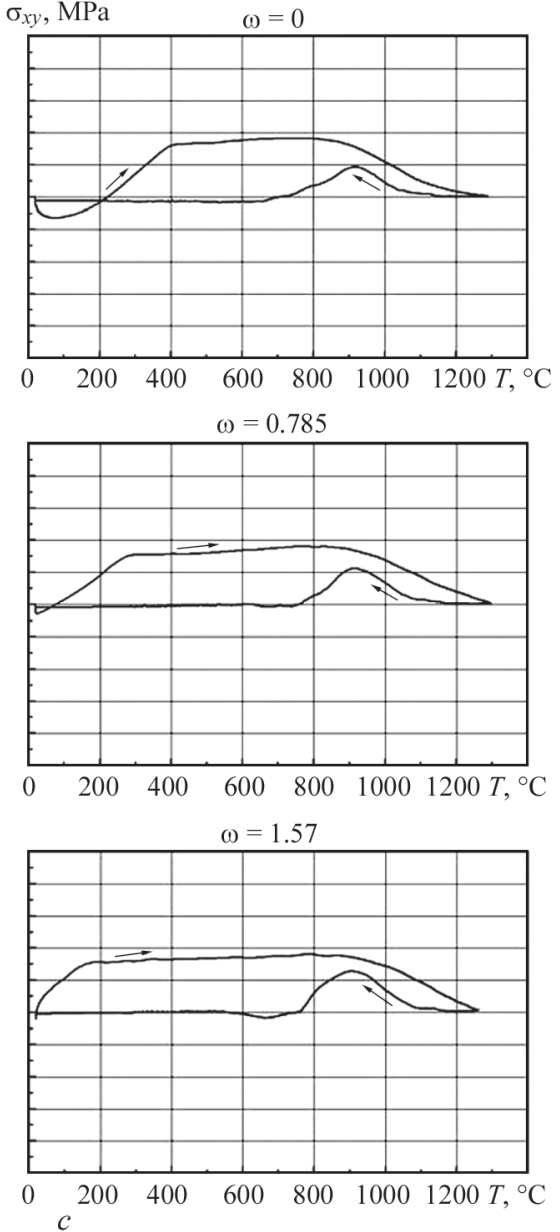

Figure 5. Kinetics of stress change: $a-\sigma_{x x}, b-\sigma_{y y}, c-\sigma_{x y}$ from temperature in point $2(\Delta y=0.5 \mathrm{~mm})$ taking into account anisotropy of $\lambda$ for different $\omega$

If $\sigma<0$, then the slip plane is parallel to axis $z$ and at $\varphi= \pm \pi / 4$ angle to axis $x$.

Corresponding calculations with initial data in Figure 7 and condition (6)-(8) were made in scope of given work.

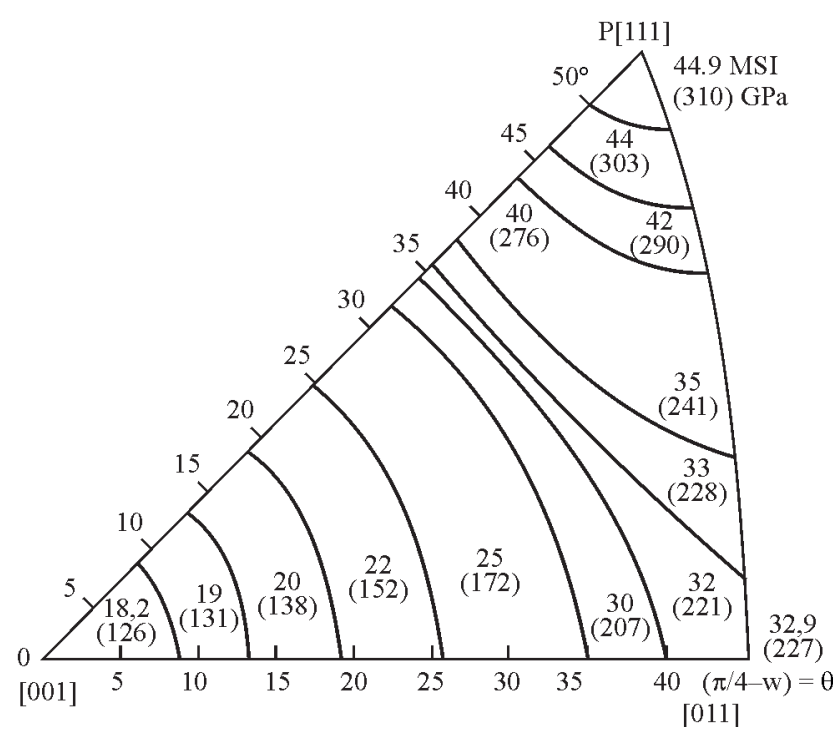

Figure 6. Dependence of elasticity modulus $E$ on angles $\omega$ and $\rho$ in single crystal of nickel alloy PWA 1480 on data [5] at $T=20^{\circ} \mathrm{C}$
Data given in Table 2 demonstrate the level of indicated effect of $\sigma_{y}$ anisotropy on the rate of plastic deformations $\Delta \varepsilon_{x x}^{p}{ }^{y}$ in the BTI-II zone.

Thus, effect of anisotropy of each of mentioned parameters $\lambda, \alpha, E$ and $\sigma_{\mathrm{y}}$ on kinetics of plastic deformations in the BTI-II zone was considered. A variant of simultaneous effect of anisotropy of all these parameters (Table 2) was also studied.

It can be seen that in this case overlaying of effect of separate parameters is also possible, that promotes

Table 3. Value of Young's modulus and characteristics of shortterm strength of $\mathrm{ZhS}-32$ alloy [6]

\begin{tabular}{|c|c|c|c|c|}
\hline$T, \mathrm{~K}$ & $<\mathrm{hkl}>$ & $E, \mathrm{GPa}$ & $\sigma_{0.2}, \mathrm{MPa}$ & $\sigma_{\mathrm{t}}, \mathrm{MPa}$ \\
\hline \multirow{3}{*}{293} & $<001>$ & 112 & 1005 & 1315 \\
\cline { 2 - 5 } & $<011>$ & 174 & 820 & - \\
\cline { 2 - 5 } & $<111>$ & 272 & 1330 & 1650 \\
\hline \multirow{3}{*}{1073} & $<001>$ & 97 & 1045 & 1310 \\
\cline { 2 - 5 } & $<011>$ & 161 & 815 & - \\
\cline { 2 - 5 } & $<111>$ & 247 & 1145 & 1295 \\
\hline \multirow{3}{*}{1173} & $<001>$ & 82 & 865 & 1035 \\
\cline { 2 - 5 } & $<011>$ & 144 & 795 & - \\
\cline { 2 - 5 } & $<111>$ & 222 & 990 & 1025 \\
\hline \multirow{3}{*}{1273} & $<001>$ & 76 & 620 & 755 \\
\cline { 2 - 5 } & $<011>$ & 121 & 535 & - \\
\cline { 2 - 5 } & $<111>$ & 214 & 650 & 735 \\
\hline
\end{tabular}


for significant rise of the rate of plastic deformations in the BTI-II.

\section{Conclusion}

One of the significant technological problems in welding heating of the parts (plates) from heat-resistant nickel alloys of ZhS-32 type, having single crystal structure, is prevention of hot crack formation in the brittleness temperature interval.

Available experience of welding of similar details from polycrystalline alloy of close type [9] shows that the main reason of appearance of indicated defects in fusion welding of such alloys is the BTI-II presence in zone of relatively moderate temperatures and related with welding heating thermal-deformation cycles, which develop in the BTI-II sufficiently high rates of plastic deformation development. It is known fact that the single crystals of heat-resistant alloys can be characterized by series of higher properties in comparison with similar alloys of polycrystalline structure.

Research, carried in the present work, shows that anisotropy of the properties of single crystal promotes for rise of plastic deformation rate in the BTI-II temperature interval in comparison with isotropic alloy of such type (having polycrystalline structure).

The rate of growth of longitudinal plastic deformations in single crystal in the BTI-II temperature interval can approximately $2.0-2.5$ times exceed that in polycrystalline variant of alloy, that obviously should be taken into account in development of corresponding structures and technology of their manufacture.

1. Ashkenazi, E.K., Ganov, E.V. (1972) Anisotropy of structural materials: Refer. Book. Leningrad: Mashinostroenie.

2. Ueda, J., Murakawa, H., Nakacho, K. et al. (1995) Establishment of computation welding mechanics. Transact. of JWRI, 24(2), 73-86.

3. Makhnenko, V.I. (1976) Computational methods for investigation of welding stress and strain kinetics. Kiev: Naukova Dumka.

4. Makhnenko, V.I., Velikoivanenko, E.A., Pochinok, V.E. (1999) Numerical methods of the predictions of welding stresses and distortions. Welding and Surfacing Reviews, 13, Pt 1, 147.

5. Goldschmidt, D. (1994) Einkristalline Gasturbinenschaufeln aus Nickelbasis-Legierungen. Materialwissenschaft und Werkstofftechnik, 25, 373-382.

6. Golubovsky, E.R., Svetlov, I.L. (2005) Static and cyclic strength of single crystals of heat-resistant nickel alloys. In: Abstr. of Pap. of Int. Sci.-Techn. Conf. on Dynamics, Strength and Life of Machines and Constructions (Ukraine, Kyiv, 1-4 Nov. 2005). Kyiv: IPS, Vol. 1, 96-97.

7. Rabotnov, Yu.N. (1963) Resistance of materials. Moscow: Fizmatgiz.

8. Birger, I.A., Mavlyutov, R.R. (1986) Resistance of materials: Manual. Moscow: Nauka.

9. Makhnenko, V.I., Savchenko, V.S., Yushchenko, K.A. et al. (1993) Influence of physical characteristics of cast nickel al-
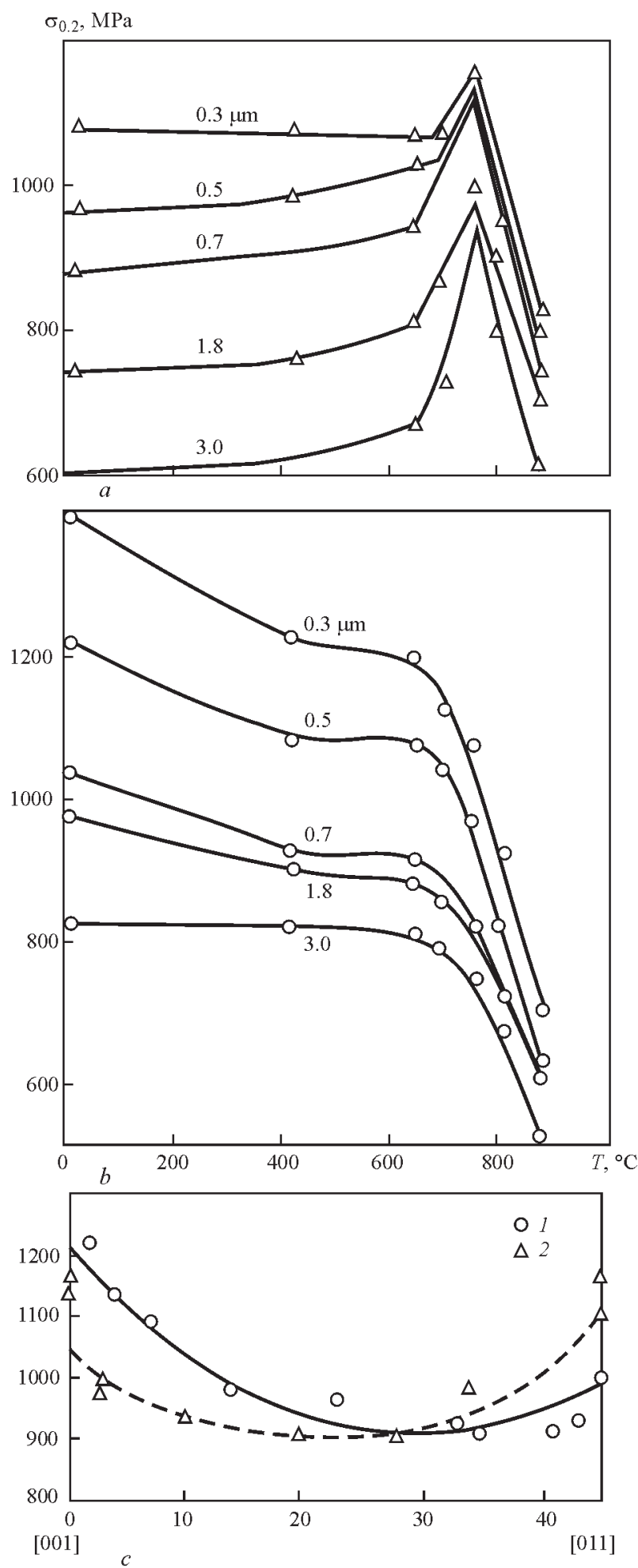

Figure 7. Yield strength of PWA 1480 alloy in sections [001] (a) and [111] $(b)$ at different size of $\gamma^{\prime}$-phase depending on temperature $(a, b)$ and tensile and compression yield strength for $593{ }^{\circ} \mathrm{C}$ depending on angle $\Theta=(\pi / 4)-\omega$ at $\rho=0(c)$ [5] (1 - tensile load; 2 - compression load)

loys on development of thermodeformation processes in fusion welding. Avtomatich. Svarka, 11, 6-9.

Received 23.05.2016 